\title{
Efficient routing with reduced routing overhead and Retransmission of Manet
}

\author{
Megha Sharma, Shivani Rohilla, Manish Bhardwaj \\ Department of Computer science and Engineering, SRM University, NCR Campus, Modinagar, Ghaziabad, India.
}

Email address:

Megha.tech09@gmail.com (M. Sharma),shivani.engineer@gmail.com (S. Rohilla), aapkaapna13@gmail.com (M. Bhardwaj)

\section{To cite this article:}

Megha Sharma, Shivani Rohilla, Manish Bhardwaj. Efficient Routing with Reduced Routing Overhead and Retransmission of Manet. American Journal of Networks and Communications. Special Issue: Ad Hoc Networks. Vol. 4, No. 3-1, 2015, pp. 22-26. doi: 10.11648/j.ajnc.s.2015040301.15

\begin{abstract}
Adhoc network is a collection of mobile nodes that dynamically form a temporary network and are capable of communicating with each other without the use of a network infrastructure. In manet nodes can change location and configure itself, the mobility of nodes causes continuous link breakage due to which frequent path failure occur and route discovery is required. The fundamental construction for a route discoveries is broadcasting in which the receiver node blindly rebroadcast the first received route request packet unless it has route to the destination. The routing overhead associated with route discovery is very high which leads to poor packet delivery ratio and a high delay to be victorious this type of routing overhead we are proposing the new technique using NCPR. To intended NCPR method is used to determine the rebroadcast order and obtain the more precise additional coverage ratio by sensing neighbor coverage knowledge. We can also define connectivity factor to provide node density adaptation. By combining the additional coverage ratio and connectivity factor, rebroadcast probability is determined. The approach can signify improvement in routing performance and decrease the routing overhead by decreasing the number of retransmission.
\end{abstract}

Keywords: Mobile Adhoc Networks, Neighbor Coverage Network Connectivity, Probabilistic Rebroadcast, Routing Overhead

\section{Introduction}

A manet is a type of network where topology changes randomly and no infrastructure is available for the network to establish an active connection .Mobile adhoc network consist of a collection of mobile nodes which can move freely. These nodes can be dynamically self -organized into arbitrary topology networks without a fixed infrastructure .one of the fundamental challenge of manet is the design of dynamic routing protocols with good performance and less overhead. Many routing protocols, such as adhoc on demand distance vector routing (AODV) [1] and dynamic source routing (DSR)[2] have been proposed, these protocol performance not constant, it is more ever variable due high mobility, network load and network size Whereas AODV creates no extra traffic for communication along existing links. The adhoc on demand distance vector routing algorithm is a routing protocol designed for adhoc mobile networks. AODV is capable of both unicast and multicast routing. It is an on demand algorithm, meaning that it builds routes between nodes only as desired by source nodes. It maintains these routes as long as they are needed by the sources [19]. the dynamic source routing(DSR), it is simple and efficient routing protocol designed specifically for use in multi hop wireless adhoc networks of mobile nodes.DSR allows the network to be completely self-organizing and self-configuring, without the need for any existing network infrastructure or administration. The above two protocols are on-demand routing protocols and they could improve the scalability of manet by limiting the routing overhead of routing protocols and reduce the packet delivery ratio and increasing the end to end delay [4].

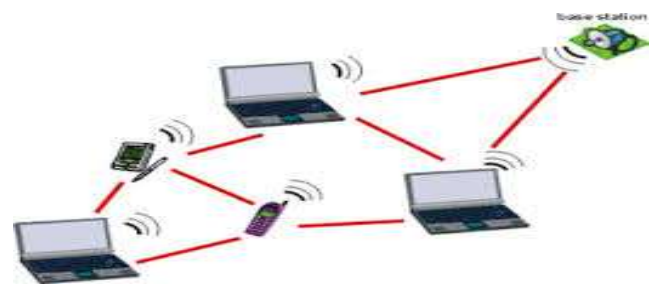

Figure 1. Multi hop ad hoc network 
The main announcement of this paper as:

- We propose a novel scheme to calculate the rebroadcast delay. The rebroadcast delay is to determine the forwarding order. The node which has more common neighbors with the previous node has the lower delay. If this node rebroadcast delay enables the information that the nodes have transmitted the packet spread to more neighbors.

- We also propose a novel scheme to calculate the rebroadcast probability the scheme considers the information about the uncovered neighbors (UCN), connectivity metric and local node density to calculate the rebroadcast probability is composed of two parts: a) additional coverage ratio, which is the ratio of the number of nodes that should be covered by a single broadcast to the total number of neighbors. b) Connectivity factor which reflects the relationship of network connectivity and the number of neighbor of a given node.

\subsection{Study the Paper Scope}

Since limiting the number of rebroadcast can effectively optimize the broadcasting and the neighbor knowledge methods perform better than the area-based ones and the probability based ones then we propose a neighbor coverage based probability (NCPR).

\subsection{Additional Coverage Ratio}

In order to effectively exploit the neighbor coverage knowledge, we need a novel rebroadcast delay to determine the rebroadcast order and then we can obtain a more precise additional coverage ratio.

\subsection{Connectivity Factor}

In order to keep the network connectivity and reduce the redundant retransmissions, we need a metric named connectivity factor to determine how many neighbors should receive the RREQ packet.

\section{Literature Review}

Broadcasting is an effective mechanism for route discovery, But the routing overhead associated with the broadcasting Can be quite large, especially in high dynamic networks [9]. Ni et al. [5] studied the broadcasting protocol analytically And experimentally, and showed that the rebroadcast is Very costly and consumes too much network resource. The Broadcasting incurs large routing overhead and causes many problems such as redundant retransmissions, contentions, and collisions [5]. Thus, optimizing the broadcasting.

In route discovery is an effective solution to improve the routing performance. Haas et al. [10] proposed a gossip based approach, where each node forwards a packet with a Probability. They showed that gossip-based approach can save up to 35 percent overhead compared to the flooding. However, when the network density is high or the traffic load is heavy, the improvement of the gossip-based approach is limited [9]. Kim et al. [8] proposed a probabilistic broadcasting scheme based on coverage area and neighbor confirmation. This scheme uses the coverage. Area to set the rebroadcast probability, and uses the neighbor confirmation to guarantee reach ability. Peng and $\mathrm{Lu}$ [11] proposed a neighbor knowledge scheme named Scalable Broadcast Algorithm (SBA). This scheme determines the rebroadcast of a packet according to the fact whether this rebroadcast would reach additional nodes. Abdulai et al. [12] proposed a Dynamic Probabilistic Route Discovery (DPR) scheme based on neighbor coverage. In This approach, each node determines the forwarding Probability according to the number of its neighbors and the set of neighbors which are covered by the previous Broadcast. This scheme only considers the coverage ratio by the previous node, and it does not consider the neighbours receiving the duplicate RREQ packet. Thus, there is a room of further optimization and extension for the DPR protocol.

Several robust protocols have been proposed in recent years besides the above optimization issues for broadcasting.

Chen et al. [13] proposed an AODV protocol with Directional Forward Routing (AODV-DFR) which takes the Directional forwarding used in geographic routing into AODV protocol. While a route breaks, this protocol can automati cally find the next-hop node for packet forwarding.

Keshavarz-Haddad et al. [14] proposed two deterministic Timer-based broadcast schemes: Dynamic Reflector Broadcast (DRB) and Dynamic Connector-Connector Broadcast (DCCB). They pointed out that their schemes can achieve full reach ability over an idealistic lossless MAC layer, and for the situation of node failure and mobility, their schemes are robustness. Stann et al. [15] proposed a Robust Broadcast Propagation (RBP) protocol to provide near-perfect reliability for flooding in wireless networks, and this protocol also has a good efficiency. They presented a new perspective for broadcasting: not to make a single broadcast more efficient but to make a single broadcast more reliable, which means by reducing the frequency of upper layer invoking flooding to improve the overall performance of flooding. In our protocol, we also set a deterministic rebroadcast delay, but the goal is to make the dissemination of neighbor knowledge much quicker.

\section{Analysis of Problem}

In mobile adhoc network nodes are moving continuously due to node mobility in manet frequent link breakages may lead to frequent path failures and route. They broadcast a route request (RREQ) packet to the network and the broadcasting induces excessive redundant retransmission of (RREQ) packet and causes the broadcast storm problem [5], which leads to a considerable number of packet collisions, especially in dense networks.

The broadcast storm problem:

A forwarding order approach to perform broadcast is by flooding .a host, on receiving a broadcast packet for the first time, has the obligation to rebroadcast the packet. Since, this costs $n$ transmission in a Manet of $n$ hosts. 
- Redundancy: when a mobile host broadcasts a packet if many of its neighbors decide to rebroadcast a broadcast packet to its neighbor, all of its neighbor might already have heard the packet.

- Contention: After a mobile host broadcasts a packet if many of its neighbor decide to rebroadcast the packet, these transmission may severely contend with each other.

In flooding, a broadcast packet is forwarded by every node in the network exactly once. The broadcast packet is absolutely to be received by every node in the network providing there is no packet loss caused be collision in the MAC layer and there is no high speed movement of nodes during the broadcast process. Figure show a network w th six nodes .when node $\mathrm{v}$ broadcast a packet all neighboring nodes $. \mathrm{u}, \mathrm{w}, \mathrm{x}$ and $\mathrm{y}$ receive the packet due to the broadcast nature of wireless communication media. All neighbors will then forward the packet to each other. Apparently, the two transmissions from nodes $\mathrm{u}$ and $\mathrm{x}$ are unnecessary.

\subsection{Proposed Work}

To calculate the rebroadcast delay and rebroadcast probability of the proposed protocol . using the upstream coverage ratio of an RREQ packet received from the previous node to calculate the rebroadcast delay and use the additional coverage ratio of the RREQ packet and the connectivity factor to calculate rebroadcast probability in our protocol, which requires that each node needs its 1- hop neighborhood information.

\subsection{Uncovered Neighbor Set And Rebroadcast Delay}

The node receives the RREQ packet from its earlier node $\mathrm{s}$, to use the neighbor list in the RREQ packet to estimate how many its neighbors have been not covered by the RREQ packet from $\mathrm{s}$. the node ni has more neighbor not covered by the RREQ packet from the source and the RREQ packet form can reach more additional neighbor nodes when node ni rebroadcast the RREQ packet . To quantify of the uncovered neighbor (UCN) set $\mathrm{u}$ (ni) of node.

$$
\mathrm{U}(\text { ni) }=\mathrm{N}(\text { ni) }-[\mathrm{N}(\mathrm{ni}) \cap \mathrm{N}(\mathrm{s})]-\{\mathrm{s}\}
$$

The rebroadcast delay $\mathrm{Td}(\mathrm{Ni})$ of node $\mathrm{Ni}$.

$$
\begin{aligned}
& \mathrm{Tp}(\text { ni) }=1-|\mathrm{N}(\mathrm{s}) \cap \mathrm{N}(\mathrm{ni})| /|\mathrm{N}(\mathrm{s})| \\
& \mathrm{Td}(\mathrm{Ni})=\max \text { delay } * \mathrm{Tp}(\text { ni })
\end{aligned}
$$

\subsection{Neighbor Knowledge and Rebroadcast Probability}

The node which has a larger rebroadcast delay may listen to RREQ packets from the nodes which have lower one. For example if node ni receives a duplicate RREQ packet from its neighbor nj, it knows that how many its neighbors have been covered by the RREQ packet from nj.thus node ni could further adjust its UCN set according to the neighbor list in the RREQ packet from nj.

$$
\mathrm{U}(\mathrm{Ni})=\mathrm{u}(\mathrm{ni})-[\mathrm{u}(\mathrm{ni}) \cap \mathrm{n}(\mathrm{nj})]
$$

Now we study how to use the final UCN set to set the rebroadcast probability.

$$
\begin{gathered}
\text { Ra (ni) }=\mid \mathrm{U}(\text { ni) }|/| \mathrm{N}(\text { ni }) \mid \\
\text { Fc (ni) }=\mathrm{Nc} / \mid \mathrm{N}(\text { ni) } \mid
\end{gathered}
$$

$\mathrm{Nc}=5.1774 \log \mathrm{n}$, the $\mathrm{n}$ is the number of nodes in the network $\mid \mathrm{N}($ ni) $\mid>\mathrm{Nc}, \mathrm{fc}($ ni) $)<1$ in the dense area of the network. $|\mathrm{N}(\mathrm{ni})|<\mathrm{Nc}, \mathrm{fc}(\mathrm{ni})>1$ in the sparse area of the network, then node ni should forward the RREQ packet in order to approach network connectivity .combining the additional coverage ratio and connectivity factor, to obtain the rebroadcast probability pre(ni) of node ni :

$$
\operatorname{Pre}(\text { ni })=f \mathrm{c}(\text { ni).ra(ni) }
$$

Where, if the pre(ni) is $>1$, to set the pre (ni) to 1 .

Algorithm description:

The formal description of the Neighbor Coverage-based Probabilistic Rebroadcast for reducing routing overhead in route discovery is shown in Algorithm 1.

Algorithm 1. NCPR

Definitions:

RREQ v: RREQ packet received from node $\mathrm{v}$.

Rv.id: the unique identifier (id) of RREQ $v$.

$\mathrm{N}(\mathrm{u})$ : Neighbor set of node $\mathrm{u}$.

$\mathrm{U}(\mathrm{u}, \mathrm{x})$ : Uncovered neighbors set of node $\mathrm{u}$ for RREQ whose

id is $\mathrm{x}$.

Timer( $\mathrm{u}, \mathrm{x})$ : Timer of node $\mathrm{u}$ for RREQ packet whose id is $\mathrm{x}$.

Note that, in the actual implementation of NCPR protocol, every different RREQ needs a UCN set and a Timer.\}

1: if ni receives a new RREQs from $s$ then

2: \{Compute initial uncovered neighbors set U(ni,Rs,id) for RREQ s: $\}$

3: $\mathrm{U}($ ni, R, :id $)=\mathrm{N}($ ni $)-[\mathrm{N}($ ni $) \backslash \mathrm{N}(\mathrm{s}])-(\mathrm{s})$

4: $\{$ Compute the rebroadcast delay $\mathrm{Td}(\mathrm{ni}):\}$

5: $\mathrm{Tp}(\mathrm{ni})=1-|\mathrm{N}(\mathrm{s}) \cap \mathrm{N}(\mathrm{ni})| /|\mathrm{N}(\mathrm{s})|$

6: $\mathrm{Td}(\mathrm{Ni})=\max$ delay $* \mathrm{Tp}(\mathrm{ni})$

7: Set a Timer(ni,Rs,id) according to Td(ni)

8: end if

9:

10: while ni receives a duplicate RREQ $\mathrm{j}$ from nj before

Timer (Ni, Rs, id) expires do

11: $\{$ Adjust U (ni, Rs, id) :\}

12: $U($ ni,Rs,id $)=($ ni, Rs,id $)-[U(n i, R, ; i d) \backslash N(n j)]$

13: discard(RREQ j)

14: end while

15:

16: if Timer (ni,Rs,id) expires then

17: $\{$ Compute the rebroadcast probability Pre(ni):\}

18: $\mathrm{Ra}($ ni $)=\mid \mathrm{U}($ ni, Rs, id $)|/| \mathrm{N}(\mathrm{ni}) \mid$

19: $\mathrm{Fc}(\mathrm{ni})=\mathrm{Nc} /|\mathrm{N}(\mathrm{ni})|$

20: $\operatorname{Pre}(n i)=f c(n i) \cdot r a(n i)$

21: if Random $(0,1)<=\operatorname{Pre}(\mathrm{ni})$ then

22: broadcast (RREQs)

23: else 
24: discard (RREQs)

25: end if

26: end if

\section{Result and Experiment}

This result will show the comparison of AODV and NCPR using the term throughput, packet delivery, end to end delay .

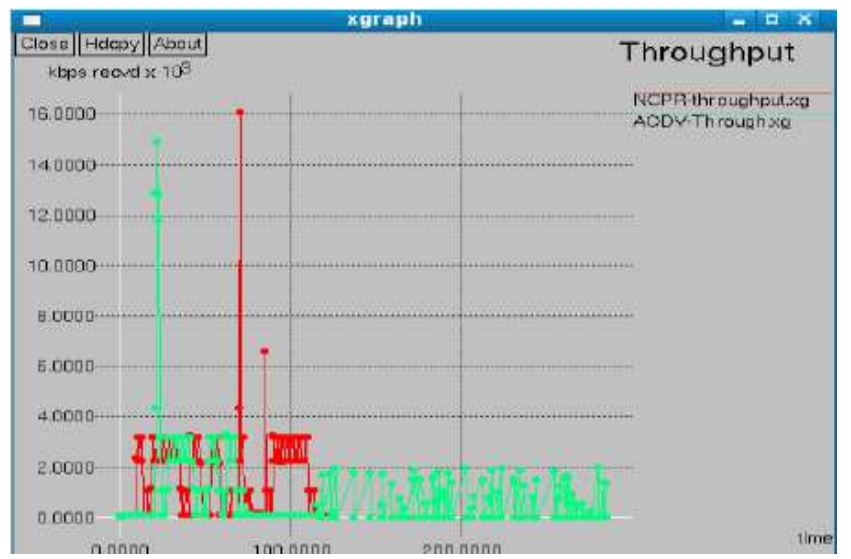

Fig (3). show the throughput

Throughput between the time and packet sent kbps. This graph show comparison of throughput between the AODV and NCPR protocol the AODV protocol gives the efficient throughput at the end of the packet delivery. But the NCPR protocol gives the most efficient throughput in starting itself.

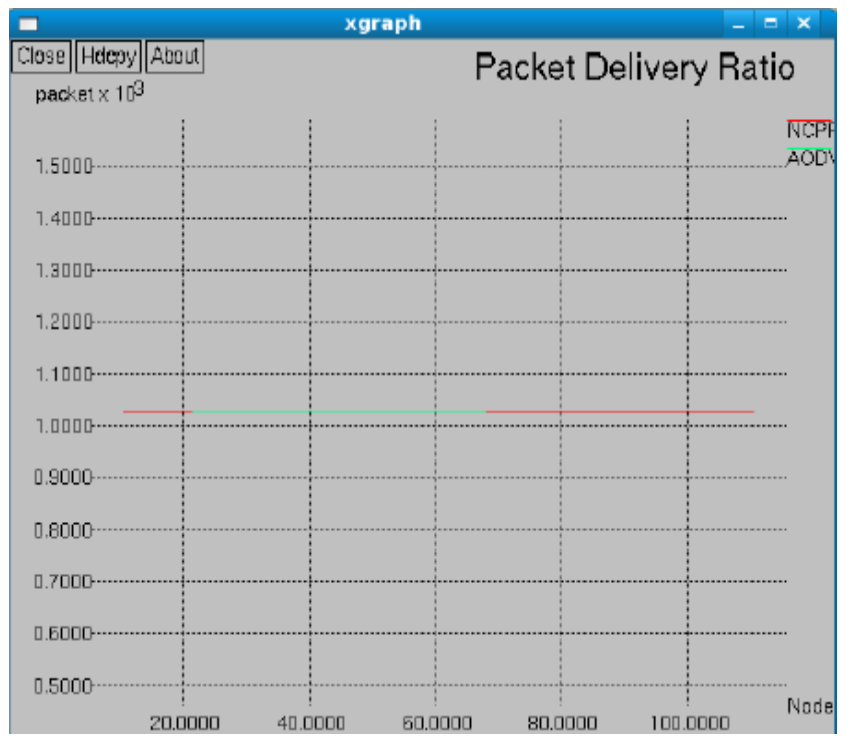

Fig (4). show the packet delivery ratio

Packet delivery ratio comparison between AODV and NCPR protocol. The packet delivery ratio means plot the graph between node and average packet delivery .it shows that PDR of AODV is efficient.

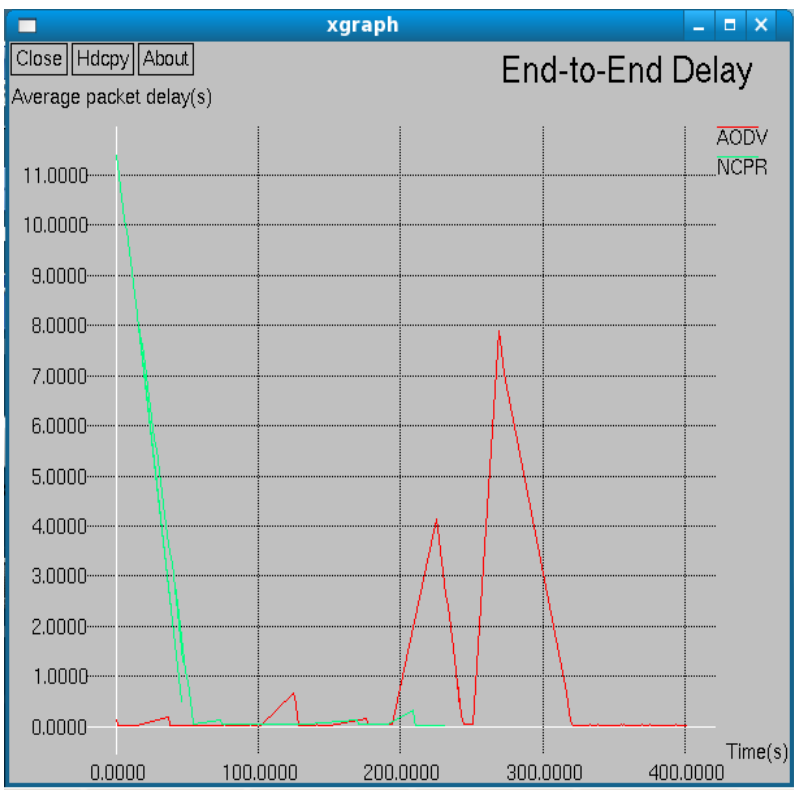

Fig (5). shows the end to and delay

The end to end delay of NCPR is high at initial time interval but when time increases as compared to AODV. In average packet delay of NCPR protocol is more efficient than AODV protocol packet delay

\section{Conclusion}

In this paper introduced probabilistic rebroadcast mechanism based on neighbor coverage to reduce the routing overhead in manet.the Paper to be constructed on the basis of good performance of the network is in high density or the traffic load is high. We proposed a new scheme to dynamically calculate the rebroadcast delay, which is used to determine the forwarding order and more exploit the neighbor coverage knowledge. Because of less redundant rebroadcast the proposed protocol mitigates the network collision and contention, so as to increase the packet delivery ratio and throughput and decrease the average end to end delay.

\section{Reference}

[1] C. Perkins, E. Belding-Royer, and S. Das, Ad Hoc On-Demand Distance Vector (AODV) Routing, IETF RFC 3561, 2003.

[2] D. Johnson, Y. Hu, and D. Maltz, The Dynamic Source Routing Protocol for Mobile Ad Hoc Networks (DSR) for IPv4, IETF RFC 4728, vol. 15, pp. 153-181, 2007.

[3] H. AlAamri, M. Abolhasan, and T. Wysocki, "On Optimising Route Discovery in Absence of Previous Route Information in MANETs," Proc. IEEE Vehicular Technology Conf. (VTC), pp. 1-5, 2009.

[4] X. Wu, H.R. Sadjadpour, and J.J. Garcia-Luna-Aceves, "Routing Overhead as a Function of Node Mobility: Modeling Framework and Implications on Proactive Routing," Proc. IEEE Int'l Conf. Mobile Ad Hoc and Sensor Systems (MASS '07), pp. 1- 9, 2007. 
[5] S.Y. Ni, Y.C. Tseng, Y.S. Chen, and J.P. Sheu, "The Broadcast Storm Problem in a Mobile Ad Hoc Network," Proc. ACM/IEEE Mob iCom, pp. 151-162, 1999.

[6] A. Mohammed, M. Ould-Khaoua, L.M. Mackenzie, C. Perkins, and J.D. Abdulai, "Probabilistic Counter-Based Route Discovery for Mobile Ad Hoc Networks," Proc. Int'l Conf. Wireless Comm. And Mobile Computing: Connecting the World Wirelessly (IWCMC '09), pp. 1335-1339, 2009.

[7] B. Williams and T. Camp, "Comparison of Broadcasting Techniques for Mobile Ad Hoc Networks," Proc. ACM MobiHoc, pp. 194- 205, 2002.

[8] J. Kim, Q. Zhang, and D.P. Agrawal, "Probabilistic Broadcasting Based on Coverage Area and Neighbor Confirmation in Mobile Ad Hoc Networks," Proc. IEEE GlobeCom, 2004.

[9] J.D. Abdulai, M. Ould-Khaoua, and L.M. Mackenzie, "Improving Probabilistic Route Discovery in Mobile Ad Hoc Networks," Proc. IEEE Conf. Local Computer Networks, pp. 739-746, 2007.

[10] Z. Haas, J.Y. Halpern, and L. Li, "Gossip-Based Ad Hoc Routing," Proc. IEEE INFOCOM, vol. 21, pp. 1707-1716, 2002.

[11] W. Peng and X. Lu, "On the Reduction of Broadcast Redundancy in Mobile Ad Hoc Networks," Proc. ACM MobiHoc, pp. 129-130, 2000.

[12] J.D. Abdulai, M. Ould-Khaoua, L.M. Mackenzie, and A. Mohammed, "Neighbour Coverage: A Dynamic Probabilistic Route Discovery for Mobile Ad Hoc Networks," Proc. Int'l Symp. Performance Evaluation of Computer and Telecomm. Systems (SPECTS'08), pp. 165-172, 2008.
[13] J. Chen, Y.Z. Lee, H. Zhou, M. Gerla, and Y. Shu, "Robust Ad Hoc Routing for Lossy Wireless Environment," Proc. IEEE Conf. Military Comm. (MILCOM '06), pp. 1-7, 2006.

[14] A. Keshavarz-Haddady, V. Ribeirox, and R. Riedi, "DRB and DCCB: Efficient and Robust Dynamic Broadcast for Ad Hoc and Sensor Networks," Proc. IEEE Comm. Soc. Conf. Sensor, Mesh, and Ad Hoc Comm. and networks (SECON '07), pp. 253-262, 2007.

[15] F. Stann, J. Heidemann, R. Shroff, and M.Z. Murtaza, "RBP: Robust Broadcast Propagation in Wireless Networks," Proc. Int'l Conf. Embedded Networked Sensor Systems (SenSys '06), pp. 85-98, 2006.

[16] F. Xue and P.R. Kumar, "The Number of Neighbors Needed for Connectivity of Wireless Networks," Wireless Networks, vol. 10, no. 2, pp. 169-181, 2004.

[17] X.M. Zhang, E.B. Wang, J.J. Xia, and D.K. Sung, "An Estimated Distance Based Routing Protocol for Mobile Ad Hoc Networks," IEEE Trans. Vehicular Technology, vol. 60, no. 7, pp. 3473-3484, Sept. 2011.

[18] Y.-C. Tseng, S.-Y. Ni, Y.-S.Chen, and J.-P. Sheu, "The Broadcast Storm Problem in a Mobile Ad Hoc Network," Wireless Networks, vol. 8, nos. 2/3, pp. 153-167, Mar.-May 2002 .

[19] http://moment.cs.ucsb.edu/AODV/aodv.html

[20] http://www.cs.cmu.edu/ dmaltz/dsr.html Ambarish R. Bhuyar et al, / (IJCSIT) International Journal of Computer Science and Information Technologies, Vol. 5 (1), 2014,390-393 